TRANSACTIONS OF THE

AMERICAN MATHEMATICAL SOCIETY

Volume 351, Number 7, Pages 2607-2622

S 0002-9947(99)02408-3

Article electronically published on February 9, 1999

\title{
BROWNIAN SHEET IMAGES AND BESSEL-RIESZ CAPACITY
}

\author{
DAVAR KHOSHNEVISAN
}

\begin{abstract}
We show that the image of a 2-dimensional set under $d$-dimensional, 2-parameter Brownian sheet can have positive Lebesgue measure if and only if the set in question has positive $(d / 2)$-dimensional Bessel-Riesz capacity. Our methods solve a problem of J.-P. Kahane.
\end{abstract}

\section{INTRODUCTION}

Consider two independent $d$-dimensional Brownian motions $X \triangleq(X(t) ; t \geqslant 0)$ and $Y \triangleq(Y(t) ; t \geqslant 0)$. Let $E_{1}$ and $E_{2}$ denote two disjoint compact subsets of $[0, \infty[$. By [7, Proposition 4, Chapter 16.6],

$$
\mathbb{P}\left(X\left(E_{1}\right) \cap X\left(E_{2}\right) \neq \varnothing\right)>0 \Longleftrightarrow \mathbb{P}\left(\operatorname{Leb}_{d}\left(X\left(E_{1}\right)+Y\left(E_{2}\right)\right)>0\right)>0,
$$

where Leb $_{d}$ denotes $d$-dimensional Lebesgue measure. Define additive Brownian motion $Z \triangleq(Z(s, t) ; s, t \geqslant 0)$ by

$$
Z(s, t) \triangleq X(s)+Y(t) .
$$

Thus, Eq. (1.1) can be rewritten as

$$
\mathbb{P}\left(X\left(E_{1}\right) \cap X\left(E_{2}\right) \neq \varnothing\right)>0 \Longleftrightarrow \mathbb{P}\left(\operatorname{Leb}_{d}\left(Z\left(E_{1} \times E_{2}\right)\right)>0\right)>0 .
$$

Consequently, self-intersection problems for a single Brownian motion naturally translate themselves to problems about the Cartesian product $E_{1} \times E_{2}$ and its image under the $(2, d)$-random field $Z$; we follow [1] for notation on $(N, d)$ fields.

The goal of this paper is to provide an analytical condition on $E_{1} \times E_{2}$ which is equivalent to (1.1). This solves a problem of J.-P. Kahane. We will actually be concerned with a more intricate problem involving the Brownian sheet. The aforementioned problem is a simple consequence of the methods employed in this paper.

To explain our results, we begin with notation and definitions which we will use throughout the paper. Any $s \in \mathbb{R}^{k}$ is written coordinatewise as $s=\left(s^{(1)}, \cdots, s^{(k)}\right)$. We will use the sup norm. That is, for all integers $k$ and all $x \in \mathbb{R}^{k}$,

$$
|x| \triangleq \max _{1 \leqslant i \leqslant k}\left|x^{(i)}\right| \text {. }
$$

Received by the editors September 23, 1997 and, in revised form, June 11, 1998.

1991 Mathematics Subject Classification. Primary 60J45; Secondary 60G15.

Key words and phrases. Capacity, Brownian sheet, additive Brownian motion, multi-parameter martingales.

Research supported by grants from the National Science Foundation and the National Security Agency.

(C)1999 American Mathematical Society 
Typographically, we shall single out the special case when $s \in[0, \infty[2$. In this case, we write $\mathbf{s},|\mathbf{s}|$, etc. for $s,|s|$, etc.; $s$ will denote 2 -dimensional time and we wish to emphasize its temporal nature by emboldening it.

For any compact set $E \subset\left[0, \infty\left[{ }^{2}\right.\right.$, we let $\mathcal{P}(E)$ denote the collection of all probability measures on $E$. For any such $E$ and for every $\beta>0$, define the $\beta$-energy of $\sigma \in \mathcal{P}(E)$ by:

$$
\mathcal{E}_{\beta}(\sigma) \triangleq \iint|\mathbf{s}-\mathbf{t}|^{-\beta} \sigma(d \mathbf{s}) \sigma(d \mathbf{t}) .
$$

The $\beta$-capacity of $E$ is defined by

$$
\operatorname{Cap}_{\beta}(E) \triangleq\left[\inf _{\sigma \in \mathcal{P}(E)} \mathcal{E}_{\beta}(\sigma)\right]^{-1} \text {. }
$$

Due to its connections with Riesz kernels and Bessel potentials, the above can aptly be called the Bessel-Riesz capacity; cf. [12] for a nice discussion of the latter objects.

In the notation of [1], we let $B \triangleq\left(B(\mathbf{s}) ; \mathbf{s} \in\left[0, \infty\left[^{2}\right)\right.\right.$ denote the $(2, d)$-Brownian sheet. That is, $B$ is a $d$-dimensional Gaussian random field, indexed by $\left[0, \infty\left[{ }^{2}\right.\right.$ with the following mean/covariance properties: for all $1 \leqslant i, j \leqslant d$ and all $\mathbf{s}, \mathbf{t} \in\left[0, \infty\left[^{2}\right.\right.$,

$$
\begin{aligned}
\mathbb{E}\left[B^{(i)}(\mathbf{s})\right] & =0, \\
\mathbb{E}\left[B^{(i)}(\mathbf{s}) B^{(j)}(\mathbf{t})\right] & =\mathbb{1}\{i=j\}\left(s^{(1)} \wedge t^{(1)}\right)\left(s^{(2)} \wedge t^{(2)}\right) .
\end{aligned}
$$

Above and throughout, $\mathbb{1}\{\cdots\}$ denotes the indicator function of whatever is in the

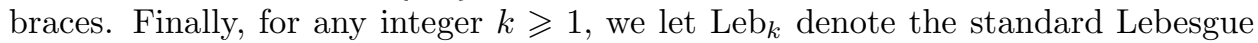
measure over $\mathbb{R}^{k}$.

The goal of this paper is to prove a quantitative version of the following:

Theorem 1.1. Suppose $E \subset\left[0, \infty\left[^{2}\right.\right.$ is compact. Then

$$
\mathbb{P}\left(\operatorname{Leb}_{d}(B(E))>0\right)>0 \Longleftrightarrow \operatorname{Cap}_{d / 2}(E)>0
$$

A simplified version of our proof of Theorem 1.1 also solves the problem of Kahane; cf. Theorem 8.2 below for a precise statement.

Clearly, Theorem 1.1 is equivalent to the following:

$$
\mathbb{E}\left[\operatorname{Leb}_{d}(B(E))\right]>0 \Longleftrightarrow \operatorname{Cap}_{d / 2}(E)>0 .
$$

Using the methods of $[7$, Chapter 6$]$, it is not difficult to find partial conditions for the strict positivity of $\mathbb{E}\left[\operatorname{Leb}_{d}(B(E))\right]$. In particular, one can deduce the sufficiency of the $\operatorname{Cap}_{d / 2}(E)>0$ by Fourier-analytic methods. While our proof of the aforementioned sufficiency is different, it is the necessity of $\operatorname{Cap}_{d / 2}(E)>0$ which is new (and much more difficult to prove).

Here, we develop some parabolic potential theory for the Brownian sheet, using ideas from the theory of multi-parameter martingales. Although different technical issues arise, in a companion paper (cf. [10]), together with Zhan Shi, we use methods with a similar flavor to study steady-state (or non-parabolic potential theory) for the Brownian sheet. There, the theory is more complete; see $\S 8.4$ below for some remarks on what is yet to be done.

The order of this paper is as follows: in Section 2, a quantitative version of Theorem 1.1 is presented (Theorem 2.1). Using this, we demonstrate Theorem 1.1. In Section 3, we prove the lower bound in Theorem 2.1. (This includes and implies our non-Fourier-analytic proof of the sufficiency of $\operatorname{Cap}_{d / 2}(E)>0$.) The bulk of 
the paper is in the proof of the upper bound in Theorem 2.1. The latter is done in four parts. In Section 4, we describe some notation necessary in the course of the proof the upper bound. We also discuss a few estimates and some key properties of multi-parameter martingales. In particular, it is here that we exploit the following simple though important property of the parameter set $\left[0, \infty\left[{ }^{2}\right.\right.$ : it can be totally ordered by 2 partial orders which we will call $\succ_{(1)}$ and $\succ_{(2)}$. Sections 5 and 6 contain prediction estimates for martingales related to $\succ_{(1)}$ and $\succ_{(2)}$, respectively. Finally, in Section 7, we complete the proof of the desired upper bound in Theorem 2.1. We have also included an eighth section for miscellaneous remarks and a few open problems. In particular, in $\S 8$, we state a quantitative solution to Kahane's problem mentioned earlier in this section.

To facilitate the reading of this paper, we close the Introduction with the following list of important constants and where they are defined:

$c_{1}$ defined in Eq. (2.1);

$c_{2}$ defined in Eq. (2.1);

$c_{3}$ defined in Lemma 3.1;

$c_{4}$ defined in Lemma 3.2;

$c_{5}$ defined in Lemma 5.2;

$c_{6}$ defined in Lemma 6.2 .

Acknowledgements. Many thanks are due to Yuval Peres for his encouragement. I am especially grateful to Zhan Shi for several years' worth of discussions on this subject.

\section{The quantitative Result}

For any compact set $E \subset\left[0, \infty\left[{ }^{2}\right.\right.$, define,

$$
\begin{aligned}
& c_{1}(E) \triangleq \inf (|\mathbf{t}|: \mathbf{t} \in E), \\
& c_{2}(E) \triangleq \sup (|\mathbf{t}|: \mathbf{t} \in E) .
\end{aligned}
$$

The main result of this paper is the following estimate of the parabolic potential theory for the Brownian sheet.

Theorem 2.1. Let $E \subset\left[0, \infty\left[{ }^{2}\right.\right.$ be a compact set. Suppose $c_{1}(E)>0$ and that $M>0$ is fixed. Then, for all $a \in[-M, M]^{d}$,

$$
A_{1} \operatorname{Cap}_{d / 2}(E) \leqslant \mathbb{P}(a \in B(E)) \leqslant A_{2} \operatorname{Cap}_{d / 2}(E),
$$

where

$$
\begin{aligned}
& A_{1}=\frac{A_{5}}{2} \pi^{-d}\left(c_{2}(E)\right)^{-4 d} \exp \left(-\frac{4 d M^{2}}{\left(c_{1}(E)\right)^{2}}\right), \\
& A_{2}=\frac{512}{A_{5}}\left(\frac{2}{\pi}\right)^{-d}\left(A_{3} \wedge A_{4}\right)^{-1}, \\
& A_{3}=e^{-2 d}\left(1 \wedge 2 c_{2}(E)\right)^{-d}, \\
& A_{4}=\left(1 \wedge 2^{3 / 2} c_{2}(E)\right)^{-d} \exp \left(-2 d\left(M^{2}+1\right)\right), \\
& A_{5}=\left(1 \wedge c_{1}(E)\right)^{3 d / 2} .
\end{aligned}
$$

Remarks 2.1.1. (i) It is remarkable that $A_{1}$ and $A_{2}$ only depend on $E$ through $c_{1}(E)$ and $c_{2}(E)$. Can one replace the Riesz kernel $(x, y) \mapsto|x-y|^{-\beta}$ by a Martin-type 
kernel to obtain universal constants? This can be done in one-parameter settings; see [3] for details.

(ii) Recall Frostman's theorem: if $\operatorname{dim}(E)$ denotes the Hausdorff dimension of $E$, then

$$
\operatorname{dim}(E)=\sup \left\{\beta>0: \operatorname{Cap}_{\beta}(E)>0\right\} .
$$

See [7] for details. Therefore, the (stochastic) image of $E$ under the map $B$ can cover singletons with positive probability if $\operatorname{dim}(E)>d / 2$, while it cannot do so if $\operatorname{dim}(E)<d / 2$. The well-known fact that $\mathrm{Cap}_{2}\left(\mathbb{R}_{+}^{4}\right)=0$ then shows us that Theorem 2.1 is only interesting when $d \in\{1,2,3\}$. Thus, we are in agreement with the result of [11] in stating that singletons are polar for the Brownian sheet if and only if $d \geqslant 4$; see also [9]. For a complete characterization of polar sets of the Brownian sheet, see [10].

(iii) Theorem 2.1 readily implies Theorem 1.1. In fact, by Theorem 2.1 and Fubini's theorem, if $c_{1}(E)>0$,

$$
(2 M)^{d} A_{1} \operatorname{Cap}_{d / 2}(E) \leqslant \mathbb{E}\left[\operatorname{Leb}_{d}\left(B(E) \cap[-M, M]^{d}\right)\right] \leqslant(2 M)^{d} A_{2} \operatorname{Cap}_{d / 2}(E) .
$$

Since $M>0$ is arbitrary, we see that if $c_{1}(E)>0$,

$$
\mathbb{E}\left[\operatorname{Leb}_{d}(B(E))\right]>0 \Longleftrightarrow \operatorname{Cap}_{d / 2}(E)>0
$$

This proves Theorem 1.1 when $c_{1}(E)>0$. When $c_{1}(E)=0$, let

$$
E_{n} \triangleq\left\{\mathbf{t} \in E:|\mathbf{t}| \geqslant n^{-1}\right\}
$$

Elementary properties of the set function $A \mapsto \operatorname{Cap}_{d / 2}(A)$ show that, as $n \rightarrow \infty$,

$$
\operatorname{Cap}_{d / 2}\left(E_{n}\right) \uparrow \operatorname{Cap}_{d / 2}(E) .
$$

A compactness argument finishes the proof of Theorem 1.1.

\section{Proof of THE LOWER BOUND}

From now on, we fix $E$ and $M$ as in the statement of Theorem 2.1. Since $E$ is now fixed, we will also write $c_{i}$ for $c_{i}(E)(i=1,2)$. For any $\varepsilon>0, a \in \mathbb{R}^{d}$ and all $\sigma \in \mathcal{P}(E)$, we define

$$
I_{\varepsilon}^{a}(\sigma) \triangleq \int \mathbb{1}\{|B(\mathbf{s})-a| \leqslant \varepsilon\} \sigma(d \mathbf{s}) .
$$

Lemma 3.1. Suppose $0<\varepsilon<M$ and $\sigma \in \mathcal{P}(E)$. Then, for all $a \in[-M, M]^{d}$,

$$
\mathbb{E}\left[I_{\varepsilon}^{a}(\sigma)\right] \geqslant c_{3} \varepsilon^{d}
$$

where

$$
c_{3} \triangleq\left(\frac{2}{\pi c_{2}^{2}}\right)^{d} \exp \left(-\frac{2 d M^{2}}{c_{1}^{2}}\right)
$$


Proof. Recall that $B(\mathbf{s})$ have i.i.d. Gaussian components each having mean 0 and variance $s^{(1)} s^{(2)}$. Directly evaluting Gaussian densities,

$$
\begin{aligned}
\mathbb{E}\left[I_{\varepsilon}^{a}(\sigma)\right] & =\int \mathbb{P}(|B(\mathbf{s})-a| \leqslant \varepsilon) \sigma(d \mathbf{s}) \\
& \geqslant \inf _{\mathbf{s} \in E} \mathbb{P}(|B(\mathbf{s})-a| \leqslant \varepsilon) \\
& =\inf _{\mathbf{s} \in E} \prod_{j=1}^{d} \int_{a^{(j)}-\varepsilon}^{a^{(j)}+\varepsilon} \frac{e^{-u^{2} /\left(2 s^{(1)} s^{(2)}\right)}}{\sqrt{2 \pi s^{(1)} s^{(2)}}} d u \\
& \geqslant\left(2 \pi c_{2}^{2}\right)^{-d / 2} \prod_{j=1}^{d} \int_{a^{(j)}-\varepsilon}^{a^{(j)}+\varepsilon} \exp \left(-\frac{u^{2}}{2 c_{1}^{2}}\right) d u \\
& \geqslant\left(2 \pi c_{2}^{2}\right)^{-d / 2}(2 \varepsilon)^{d} \prod_{j=1}^{d} \exp \left(-\frac{\left(a^{(j)}-\varepsilon\right)^{2} \vee\left(a^{(j)}+\varepsilon\right)^{2}}{2 c_{1}^{2}}\right) \\
& \geqslant\left(\frac{2}{\pi c_{2}^{2}}\right)^{d / 2} \varepsilon^{d} \exp \left(-\frac{\sum_{j=1}^{d}\left(a^{(j)}-\varepsilon\right)^{2} \vee \sum_{k=1}^{d}\left(a^{(k)}+\varepsilon\right)^{2}}{2 c_{1}^{2}}\right) .
\end{aligned}
$$

Using Jenssen's inequality: $(a \pm \varepsilon)^{2} \leqslant 2\left(a^{2}+\varepsilon^{2}\right)$, we obtain the desired result.

Lemma 3.2. Suppose $\varepsilon>0$ and $a \in \mathbb{R}^{d}$. Then,

$$
\mathbb{E}\left[I_{\varepsilon}^{a}(\sigma)\right]^{2} \leqslant c_{4} \varepsilon^{d} \iint\left(1 \wedge \frac{\varepsilon}{|\mathbf{t}-\mathbf{s}|^{1 / 2}}\right)^{d} \sigma(d \mathbf{s}) \sigma(d \mathbf{t}),
$$

where

$$
c_{4} \triangleq 2\left(\frac{4}{\pi}\right)^{d}\left(c_{1} \wedge 1\right)^{-3 d / 2} .
$$

Proof. First, we define two partial orders on $\left[0, \infty\left[{ }^{2}\right.\right.$. For all $\mathbf{s}, \mathbf{t} \in\left[0, \infty\left[{ }^{2}\right.\right.$, define,

$$
\begin{aligned}
& \mathbf{s} \succ_{(1)} \mathbf{t} \Longleftrightarrow s^{(1)} \geqslant t^{(1)}, \text { and } s^{(2)} \geqslant t^{(2)}, \\
& \mathbf{s} \succ_{(2)} \mathbf{t} \Longleftrightarrow s^{(1)} \geqslant t^{(1)}, \text { and } s^{(2)} \leqslant t^{(2)} .
\end{aligned}
$$

The significance of these two partial orderings is that, together, $\succ_{(1)}$ and $\succ_{(2)}$ totally order $\left[0, \infty\left[{ }^{2}\right.\right.$ in that for any $\mathbf{s}_{1}, \mathbf{s}_{2} \in\left[0, \infty\left[{ }^{2}\right.\right.$, there must exist $i, j, k \in\{1,2\}$ such that $i \neq j$ and $\mathbf{s}_{i} \succ_{(k)} \mathbf{s}_{j}$.

Now, we get on with the proof. Note that

$$
\begin{aligned}
\mathbb{E}\left[I_{\varepsilon}^{a}(\sigma)\right]^{2} & =\iint \mathbb{P}(|B(\mathbf{s})-a| \leqslant \varepsilon,|B(\mathbf{t})-a| \leqslant \varepsilon) \sigma(d \mathbf{s}) \sigma(d \mathbf{t}) \\
& \leqslant \iint \mathbb{P}(|B(\mathbf{s})| \leqslant \varepsilon,|B(\mathbf{t})| \leqslant \varepsilon) \sigma(d \mathbf{s}) \sigma(d \mathbf{t}) .
\end{aligned}
$$

We have used the unimodality of mean zero multivariate Gaussian densities. By symmetry,

$$
\mathbb{E}\left[I_{\varepsilon}^{a}(\sigma)\right]^{2} \leqslant 2 Q_{1}+2 Q_{2}
$$

where

$$
Q_{1} \triangleq \iint_{\mathbf{s} \succ(1)} \mathbb{t} \mathbb{P}(|B(\mathbf{s})| \leqslant \varepsilon,|B(\mathbf{t})| \leqslant \varepsilon) \sigma(d \mathbf{s}) \sigma(d \mathbf{t})
$$


and

$$
Q_{2} \triangleq \int_{\mathbf{s} \succ(2)} \int_{\mathbf{t}} \mathbb{P}(|B(\mathbf{s})| \leqslant \varepsilon,|B(\mathbf{t})| \leqslant \varepsilon) \sigma(d \mathbf{s}) \sigma(d \mathbf{t}) .
$$

Before proceeding with detailed analysis, let us note that if $\mathbf{s} \in E$,

$$
\begin{aligned}
\mathbb{P}(|B(\mathbf{s})| \leqslant \varepsilon) & =\prod_{j=1}^{d} \int_{-\varepsilon}^{\varepsilon} \frac{\left.e^{-u^{2} /\left(2 s^{(1)} s^{(2)}\right.}\right)}{\sqrt{2 \pi s^{(1)} s^{(2)}}} d u \\
& \leqslant\left(\frac{2}{\pi}\right)^{d / 2} c_{1}^{-d} \varepsilon^{d} .
\end{aligned}
$$

Estimation of $Q_{1}$. Suppose $\mathbf{s} \succ_{(1)} \mathbf{t}$ are both in $E$ and are fixed. Then, $\Gamma \triangleq$ $B(\mathbf{t})-B(\mathbf{s})$ is independent of $B(\mathbf{s})$ and is a $d$-dimensional Gaussian random vector with mean 0 , i.i.d. coordinates. Moreover, for all $1 \leqslant i \leqslant d$,

$$
\begin{aligned}
\operatorname{Var}\left(\Gamma^{(i)}\right) & =t^{(1)}\left(s^{(2)}-t^{(2)}\right)+t^{(2)}\left(s^{(1)}-t^{(1)}\right)+\left(s^{(2)}-t^{(2)}\right)\left(s^{(1)}-t^{(1)}\right) \\
& =s^{(2)}\left(s^{(1)}-t^{(1)}\right)+t^{(1)}\left(s^{(2)}-t^{(2)}\right) \\
& \geqslant c_{1}|\mathbf{t}-\mathbf{s}| .
\end{aligned}
$$

Hence, by independence and (3.3),

$$
\begin{aligned}
\mathbb{P}(|B(\mathbf{s})| \leqslant \varepsilon,|B(\mathbf{t})| \leqslant \varepsilon) & \leqslant \mathbb{P}(|B(\mathbf{s})| \leqslant \varepsilon,|\Gamma| \leqslant 2 \varepsilon) \\
& =\mathbb{P}(|B(\mathbf{s})| \leqslant \varepsilon) \mathbb{P}(|\Gamma| \leqslant 2 \varepsilon) \\
& \leqslant\left(\frac{2}{\pi}\right)^{d / 2} c_{1}^{d} \varepsilon^{d} \prod_{k=1}^{d} \int_{-2 \varepsilon}^{2 \varepsilon} \frac{e^{-v^{2} /\left(2 \operatorname{Var} \Gamma^{(1)}\right)}}{\sqrt{2 \pi \operatorname{Var} \Gamma^{(1)}}} d v \\
& \leqslant\left(\frac{2}{\pi}\right)^{d} c_{1}^{-3 d / 2} \varepsilon^{2 d}|\mathbf{t}-\mathbf{s}|^{-d / 2} .
\end{aligned}
$$

In conjunction with (3.3), we have the following estimate for $Q_{1}$ :

$$
Q_{1} \leqslant\left(\frac{2}{\pi}\right)^{d}\left(c_{1} \wedge 1\right)^{-3 d / 2} \varepsilon^{d} \int_{\mathbf{s} \succ(1)} \int_{\mathbf{t}}\left(1 \wedge \frac{\varepsilon}{|\mathbf{t}-\mathbf{s}|^{1 / 2}}\right)^{d} \sigma(d \mathbf{s}) \sigma(d \mathbf{t}) .
$$

Estimation of $Q_{2}$. Suppose $\mathbf{s} \succ_{(2)} \mathbf{t}$ are both in $E$ and are fixed. Define,

$$
\begin{aligned}
& \Gamma_{1} \triangleq B(\mathbf{t})-B\left(t^{(1)}, s^{(2)}\right), \\
& \Gamma_{2} \triangleq B(\mathbf{s})-B\left(t^{(1)}, s^{(2)}\right), \\
& \Gamma_{3} \triangleq B\left(t^{(1)}, s^{(2)}\right) .
\end{aligned}
$$

By checking covariances, it is not difficult to see that $\left(\Gamma_{1}, \Gamma_{2}, \Gamma_{3}\right)$ are mutually independent Gaussian random vectors with mean 0 . In particular, by the unimodality of centered multivariate Gaussian distributions,

$$
\mathbb{P}\left(\left|\Gamma_{1}+\Gamma_{2}+2 \Gamma_{3}\right| \leqslant 2 \varepsilon \mid \Gamma_{1}, \Gamma_{2}\right) \leqslant \mathbb{P}\left(\left|\Gamma_{3}\right| \leqslant \varepsilon\right), \quad \text { a.s. }
$$

Applying the triangle inequality,

$$
\begin{aligned}
\mathbb{P}(|B(\mathbf{s})| \leqslant \varepsilon,|B(\mathbf{t})| \leqslant \varepsilon) & \leqslant \mathbb{P}\left(\left|\Gamma_{1}+\Gamma_{2}+2 \Gamma_{3}\right| \leqslant 2 \varepsilon,\left|\Gamma_{1}-\Gamma_{2}\right| \leqslant 2 \varepsilon\right) \\
& \leqslant \mathbb{P}\left(\left|\Gamma_{3}\right| \leqslant \varepsilon\right) \mathbb{P}\left(\left|\Gamma_{1}-\Gamma_{2}\right| \leqslant 2 \varepsilon\right) .
\end{aligned}
$$


It remains to estimate these probabilities. Since $\Gamma_{1}-\Gamma_{2}$ and $\Gamma_{3}$ both have i.i.d. mean 0 coordinates, it suffices to estimate the coordinatewise variances. This is simple. Indeed, for all $1 \leqslant i \leqslant d$,

$$
\operatorname{Var}\left(\Gamma_{1}^{(i)}-\Gamma_{2}^{(i)}\right)=s^{(2)}\left(s^{(1)}-t^{(1)}\right)+t^{(1)}\left(t^{(2)}-s^{(2)}\right) \geqslant c_{1}|\mathbf{t}-\mathbf{s}|,
$$

and

$$
\operatorname{Var}\left(\Gamma_{3}^{(i)}\right)=t^{(1)} s^{(2)} \geqslant c_{1}^{2}
$$

Combining (3.5), (3.6) and (3.7), we arrive at the following Gaussian estimation:

$$
\begin{aligned}
& \mathbb{P}(|B(\mathbf{s})| \leqslant \varepsilon,|B(\mathbf{t})| \leqslant \varepsilon) \\
& \leqslant \prod_{j=1}^{d} \int_{-\varepsilon}^{\varepsilon} \frac{e^{-u^{2} /\left(2 \operatorname{Var} \Gamma_{3}^{(1)}\right)}}{\sqrt{2 \pi \operatorname{Var} \Gamma_{3}^{(1)}}} d u \times \prod_{k=1}^{d} \int_{-2 \varepsilon}^{2 \varepsilon} \frac{e^{-v^{2} /\left(2 \operatorname{Var}\left(\Gamma_{1}^{(1)}-\Gamma_{2}^{(1)}\right)\right)}}{\sqrt{2 \pi \operatorname{Var}\left(\Gamma_{1}^{(1)}-\Gamma_{2}^{(1)}\right)}} d v \\
& \leqslant 2^{d}\left(\frac{2}{\pi}\right)^{d} c_{1}^{-3 d / 2} \varepsilon^{2 d}|\mathbf{t}-\mathbf{s}|^{-d / 2} .
\end{aligned}
$$

Integrating over all such $\mathbf{t}, \mathbf{s}$ and using (3.3), we arrive at the following estimate for $Q_{2}$ :

$$
Q_{2} \leqslant\left(\frac{4}{\pi}\right)^{d}\left(c_{1} \wedge 1\right)^{-3 d / 2} \varepsilon^{d} \int_{\mathbf{s} \succ(2)} \int_{\mathbf{t}}\left(1 \wedge \frac{\varepsilon}{|\mathbf{t}-\mathbf{s}|^{1 / 2}}\right)^{d} \sigma(d \mathbf{s}) \sigma(d \mathbf{t}) .
$$

The above, together with (3.2), (3.4) and symmetry, implies the result.

We end this section with the proof of the lower bound in Theorem 2.1. Recall the Paley-Zygmund inequality: for any random variable $Z \geqslant 0$ with $Z \in L^{2}(d \mathbb{P})$,

$$
\mathbb{P}(Z>0) \geqslant \frac{[\mathbb{E} Z]^{2}}{\mathbb{E}\left[Z^{2}\right]}
$$

For any $\sigma \in \mathcal{P}(E)$ and all $\varepsilon>0$, we apply this to $I_{\varepsilon}^{a}(\sigma)$ in the following manner:

$$
\begin{aligned}
\mathbb{P}(\exists \mathbf{s} \in E:|B(\mathbf{s})-a| \leqslant \varepsilon) & \geqslant \mathbb{P}\left(I_{\varepsilon}^{a}(\sigma)>0\right) \\
& \geqslant \frac{\left[\mathbb{E} I_{\varepsilon}^{a}(\sigma)\right]^{2}}{\mathbb{E}\left[I_{\varepsilon}^{a}(\sigma)\right]^{2}} .
\end{aligned}
$$

By Lemmas 3.1 and 3.2 and the definition of $d / 2-$ energy of $\sigma$,

$$
\mathbb{P}(\exists \mathbf{s} \in E:|B(\mathbf{s})-a| \leqslant \varepsilon) \geqslant \frac{c_{3}^{2}}{c_{4} \mathcal{E}_{d / 2}(\sigma)} .
$$

Since $\sigma \in \mathcal{P}(E)$ is arbitrary,

$$
\mathbb{P}(\exists \mathbf{s} \in E:|B(\mathbf{s})-a| \leqslant \varepsilon) \geqslant \frac{c_{3}^{2}}{c_{4}} \operatorname{Cap}_{d / 2}(E) .
$$

Since $\mathbf{s} \mapsto B(\mathbf{s})$ has an a.s. continuous modification (cf. [1]), we can let $\varepsilon \rightarrow 0$ and use compactness to obtain

$$
\mathbb{P}(a \in B(E)) \geqslant \frac{c_{3}^{2}}{c_{4}} \operatorname{Cap}_{d / 2}(E) .
$$

The lower bound in Theorem 2.1 follows from the fact that $A_{1}=c_{3}^{2} c_{4}^{-1}$. 


\section{Proof of the UpPer Bound: PART 1}

Recalling Eq. (3.1), define for all $\mathbf{t} \in\left[0, \infty\left[^{2}\right.\right.$,

$$
\begin{aligned}
& \mathcal{F}^{00}(\mathbf{t}) \triangleq \sigma\left(B(\mathbf{r}): \mathbf{t} \succ_{(1)} \mathbf{r}\right), \\
& \mathcal{G}^{00}(\mathbf{t}) \triangleq \sigma\left(B(\mathbf{r}): \mathbf{t} \succ_{(2)} \mathbf{r}\right) .
\end{aligned}
$$

Let $\mathcal{F}^{0}(\mathbf{t})$ and $\mathcal{G}^{0}(\mathbf{t})$ be the $\mathbb{P}$-completions of $\mathcal{F}^{00}(\mathbf{t})$ and $\mathcal{G}^{00}(\mathbf{t})$, respectively. Finally, we define

$$
\begin{aligned}
& \mathcal{F}(\mathbf{t}) \triangleq \bigcap_{\mathbf{r} \succ(1)} \mathbf{t} \mathcal{F}^{0}(\mathbf{r}), \\
& \mathcal{G}(\mathbf{t}) \triangleq \bigcap_{\mathbf{r} \succ(2)} \mathbf{t} \\
& \mathcal{G}^{0}(\mathbf{r}) .
\end{aligned}
$$

The following is a routine exercise in the theory of multi-parameter martingales:

Lemma 4.1. (i) $\left\{\mathcal{F}(\mathbf{t}) ; \mathbf{t} \in\left[0, \infty\left[^{2}\right\}\right.\right.$ is a complete, right continuous filtration with respect to the partial order $\succ_{(1)}$;

(ii) $\{\mathcal{G}(\mathbf{t}) ; \mathbf{t} \in[0, \infty[2\}$ is a complete, right continuous filtration with respect to the partial order $\succ_{(2)}$;

(iii) $\left\{\mathcal{F}(\mathbf{t}) ; \mathbf{t} \in\left[0, \infty\left[^{2}\right\}\right.\right.$ satisfies (F4) of R. Cairoli and J.B. Walsh (cf. [6]) with respect to $\succ_{(1)}$;

(iv) $\left\{\mathcal{G}(\mathbf{t}) ; \mathbf{t} \in\left[0, \infty\left[^{2}\right\}\right.\right.$ satisfies (F4) of R. Cairoli and J.B. Walsh (cf. [6]) with respect to $\succ_{(2)}$.

As an important consequence of the above, we obtain:

Proposition 4.2. Suppose $Y$ is a random variable in $L^{p}(d \mathbb{P})$ for some $p>1$. Then

(i) $\mathbb{E}\left[\sup _{\mathbf{t} \in[0, \infty[2}|\mathbb{E}[Y \mid \mathcal{F}(\mathbf{t})]|^{p} \leqslant\left(\frac{p}{p-1}\right)^{2 p} \mathbb{E} Y^{p}\right.$;

(ii) $\mathbb{E}\left[\sup _{\mathbf{t} \in\left[0, \infty\left[^{2}\right.\right.}|\mathbb{E}[Y \mid \mathcal{G}(\mathbf{t})]|\right]^{p} \leqslant\left(\frac{p}{p-1}\right)^{2 p} \mathbb{E} Y^{p}$;

(iii) $\mathbf{t} \mapsto \mathbb{E}[Y \mid \mathcal{F}(\mathbf{t})]$ has an a.s. continuous modification;

(iv) $\mathbf{t} \mapsto \mathbb{E}[Y \mid \mathcal{G}(\mathbf{t})]$ has an a.s. continuous modification.

Proof. Parts (i) and (iii) are special cases of Lemma 2.2 and Proposition 2.3 of [10], respectively. The key ingredient in the proofs (in our current setting) are Lemmas 4.1(i) and 4.1(iii) above. Parts (ii) and (iv) are proved along similar lines but instead we use Lemma 4.1(ii) and (iv), respectively.

The above is the main technical result of this section. We end this section with the introduction of some notation which shall be used in the subsequent sections. For $i=1,2, \sigma \in \mathcal{P}(E), \varepsilon>0$ and $a \in \mathbb{R}^{d}$, we define

$$
J_{i} \triangleq \int_{\mathbf{s} \succ(i)} \mathbf{t}\{|B(\mathbf{s})-a| \leqslant \varepsilon\} \sigma(d \mathbf{s}) .
$$

Also, we define for all $\varepsilon>0$ and $a \in \mathbb{R}^{d}$,

$$
\mathcal{H}_{\varepsilon}^{a} \triangleq\{\mathbf{t} \in E:|B(\mathbf{t})-a| \leqslant \varepsilon / 2\} .
$$

(Note the 2 in $\varepsilon / 2$ !.) 


\section{Proof OF the UPPer Bound: PART 2}

We begin with a more or less well-known path decomposition for $B$. See [5, Lemma 2.4] for a close relative of this result.

Lemma 5.1. Fix $\mathbf{t} \in\left[0, \infty\left[{ }^{2}\right.\right.$. Then, as a process in $\mathbf{s} \succ_{(1)} \mathbf{t}$,

$$
B(\mathbf{s})=B(\mathbf{t})+\sqrt{t^{(2)}} \beta_{1}\left(s^{(1)}-t^{(1)}\right)+\sqrt{t^{(1)}} \beta_{2}\left(s^{(2)}-t^{(2)}\right)+W(\mathbf{s}-\mathbf{t}),
$$

where $\beta_{1}$ and $\beta_{2}$ are $d$-dimensional Brownian motions, $W$ is a $(2, d)$-Brownian sheet, and $\left(\beta_{1}, \beta_{2}, W\right)$ are totally independent from each other and from $\mathcal{F}(\mathbf{t})$.

Proof. We will describe a proof for the sake of completeness. To clarify the picture, we use the white noise representation of [4]. Let $\dot{B}$ denote $d$-dimensional white noise spread over $\left[0, \infty\left[^{2}\right.\right.$ (viewed either as a random distribution or an $L^{2}(d \mathbb{P})$-measure). It was observed by Cěntsov that

$$
B(\mathbf{s})=\dot{B}\left(\left[0, s^{(1)}\right] \times\left[0, s^{(2)}\right]\right) .
$$

Decompose $\left[0, s^{(1)}\right] \times\left[0, s^{(2)}\right]$ as follows:

$$
\begin{aligned}
{\left[0, s^{(1)}\right] \times\left[0, s^{(2)}\right] } & =\left[0, t^{(1)}\right] \times\left[0, t^{(2)}\right] \cup\left[0, t^{(1)}\right] \times\left[t^{(2)}, s^{(2)}\right] \\
& \cup\left[t^{(1)}, s^{(1)}\right] \times\left[0, t^{(2)}\right] \cup\left[t^{(1)}, s^{(1)}\right] \times\left[t^{(2)}, s^{(2)}\right] .
\end{aligned}
$$

Using elementary properties of $\dot{B}$, we arrive at the following, a.s.: for all $\mathbf{s} \succ_{(1)} \mathbf{t}$,

$$
\begin{aligned}
B(\mathbf{s})= & B(\mathbf{t})+\dot{B}\left(\left[t^{(1)}, s^{(1)}\right] \times\left[0, t^{(2)}\right]\right) \\
& +\dot{B}\left(\left[0, t^{(1)}\right] \times\left[t^{(2)}, s^{(2)}\right]\right)+\dot{B}\left(\left[t^{(1)}, s^{(1)}\right] \times\left[t^{(2)}, s^{(2)}\right]\right) \\
\triangleq B(\mathbf{t}) & +Q_{3}+Q_{4}+Q_{5} .
\end{aligned}
$$

Since $\dot{B}$ assigns independent mass to disjoint sets, $\mathcal{F}(\mathbf{t})$ and the entire process $\left(Q_{3}, Q_{4}, Q_{5}\right)$ are totally independent. The rest of the assertions follow from covariance calculations.

Recall Eqs. (4.1) and (4.2). The main result of this section is the following technical estimate:

Lemma 5.2. Fix $\mathbf{t} \in\left[0, \infty\left[{ }^{2}, \sigma \in \mathcal{P}(E), a \in \mathbb{R}^{d}\right.\right.$ and $\varepsilon>0$. Then, a.s.,

$$
\mathbb{E}\left[J_{1} \mid \mathcal{F}(\mathbf{t})\right] \geqslant c_{5} \mathbb{1}\left\{\mathbf{t} \in \mathcal{H}_{\varepsilon}^{a}\right\} \int_{\mathbf{s} \succ(1)}\left(1 \wedge \frac{\varepsilon}{|\mathbf{s}-\mathbf{t}|^{1 / 2}}\right)^{d} \sigma(d \mathbf{s}),
$$

where

$$
c_{5} \triangleq\left(\frac{2}{e \pi}\right)^{d}\left(1 \vee 2 c_{2}\right)^{-d / 2} .
$$

Proof. By our path decomposition (Lemma 5.1),

$$
\begin{aligned}
J_{1}=\int_{\mathbf{s} \succ(1)} \mathbf{t}\left\{\mid B(\mathbf{t})+\sqrt{t^{(2)}} \beta_{1}\left(s^{(1)}-t^{(1)}\right)+\sqrt{t^{(1)}}\right. & \beta_{2}\left(s^{(2)}-t^{(2)}\right) \\
& +W(\mathbf{s}-\mathbf{t})-a \mid \leqslant \varepsilon\} \sigma(d \mathbf{s}) .
\end{aligned}
$$


Therefore, on $\left(\mathbf{t} \in \mathcal{H}_{\varepsilon}^{a}\right)$, we a.s. have the following inequality:

$$
\begin{array}{rl}
J_{1} \geqslant \int_{\mathbf{s} \succ(1)} \mathbf{t} & \mathbb{1}\left\{\mid \sqrt{t^{(2)}} \beta_{1}\left(s^{(1)}-t^{(1)}\right)+\sqrt{t^{(1)}} \beta_{2}\left(s^{(2)}-t^{(2)}\right)\right. \\
& +W(\mathbf{s}-\mathbf{t}) \mid \leqslant \varepsilon / 2\} \sigma(d \mathbf{s}) .
\end{array}
$$

By the independence assertion of Lemma 5.1,

$$
\mathbb{E}\left[J_{1} \mid \mathcal{F}(\mathbf{t})\right] \geqslant \mathbb{1}\left\{\mathbf{t} \in \mathcal{H}_{\varepsilon}^{a}\right\} \int_{\mathbf{s} \succ(1)} \mathbb{t} \mathbb{P}\left(\left|\Gamma_{4}\right| \leqslant \varepsilon / 2\right) \sigma(d \mathbf{s}),
$$

where

$$
\Gamma_{4}=\sqrt{t^{(2)}} \beta_{1}\left(s^{(1)}-t^{(1)}\right)+\sqrt{t^{(1)}} \beta_{2}\left(s^{(2)}-t^{(2)}\right)+W(\mathbf{s}-\mathbf{t}) .
$$

Fixing $\mathbf{t}$ and $\mathbf{s} \succ_{(1)} \mathbf{t}$, it is a simple Gaussian calculation that

$$
\Gamma_{4} \stackrel{(d)}{=} \sqrt{t^{(1)}\left(s^{(2)}-t^{(2)}\right)+s^{(2)}\left(s^{(1)}-t^{(1)}\right)} \beta_{1}(1) .
$$

Eq. (5.1) implies that, a.s.,

$$
\begin{aligned}
& \mathbb{E}\left[J_{1} \mid \mathcal{F}(\mathbf{t})\right] \\
& \geqslant \mathbb{1}\left\{\mathbf{t} \in \mathcal{H}_{\varepsilon}^{a}\right\} \int_{\mathbf{s} \succ(1)} \mathbb{t} P\left(\left|\beta_{1}(1)\right| \leqslant \frac{\varepsilon}{2 \sqrt{t^{(1)}\left(s^{(2)}-t^{(2)}\right)+s^{(2)}\left(s^{(1)}-t^{(1)}\right)}}\right) \sigma(d \mathbf{s}) \\
& \geqslant \mathbb{1}\left\{\mathbf{t} \in \mathcal{H}_{\varepsilon}^{a}\right\} \int_{\mathbf{s} \succ(1)} \mathbb{t} P\left(\left|\beta_{1}(1)\right| \leqslant \frac{\varepsilon}{2 \sqrt{2 c_{2}}|\mathbf{s}-\mathbf{t}|^{1 / 2}}\right) \sigma(d \mathbf{s}),
\end{aligned}
$$

since for $\mathbf{t}, \mathbf{s}$ in question,

$$
\sqrt{t^{(1)}\left(s^{(2)}-t^{(2)}\right)+s^{(2)}\left(t^{(1)}-s^{(1)}\right)} \leqslant \sqrt{2 c_{2}}|\mathbf{s}-\mathbf{t}|^{1 / 2} .
$$

Let

$$
Q_{6} \triangleq\left(\int_{-1}^{1} \frac{e^{-v^{2} / 2}}{\sqrt{2 \pi}} d v\right)^{d}
$$

If

$$
\frac{\varepsilon}{2 \sqrt{2 c_{2}}|\mathbf{s}-\mathbf{t}|^{1 / 2}} \geqslant 1
$$

then

$$
\mathbb{P}\left(\left|\beta_{1}(1)\right| \leqslant \frac{\varepsilon}{2 \sqrt{2 c_{2}}|\mathbf{s}-\mathbf{t}|^{1 / 2}}\right) \geqslant Q_{6} .
$$

On the other hand, if (5.3) does not hold,

$$
\begin{aligned}
\mathbb{P}\left(\left|\beta_{1}(1)\right| \leqslant \frac{\varepsilon}{2 \sqrt{2 c_{2}}|\mathbf{s}-\mathbf{t}|^{1 / 2}}\right) & =\left(2 \int_{0}^{\varepsilon /\left(2 \sqrt{2 c_{2}}|\mathbf{s}-\mathbf{t}|^{1 / 2}\right)} \frac{e^{-u^{2} / 2}}{\sqrt{2 \pi}} d u\right)^{d} \\
& \geqslant\left(4 e \pi c_{2}\right)^{-d / 2} \varepsilon^{d}|\mathbf{s}-\mathbf{t}|^{-d / 2} .
\end{aligned}
$$

Therefore, Eq. (5.2) implies that, a.s.,

$$
\begin{aligned}
\mathbb{E}\left[J_{1} \mathcal{F}(\mathbf{t})\right] & \geqslant \mathbb{1}\left\{\mathbf{t} \in \mathcal{H}_{\varepsilon}^{a}\right\} \int_{\mathbf{s} \succ(1)}\left\{Q_{6} \wedge\left(\frac{\varepsilon^{2}}{4 e \pi c_{2}|\mathbf{s}-\mathbf{t}|}\right)^{d}\right\} \sigma(d \mathbf{s}) \\
& \geqslant Q_{7} \mathbb{1}\left\{\mathbf{t} \in \mathcal{H}_{\varepsilon}^{a}\right\} \int_{\mathbf{s} \succ(1)}\left(1 \wedge \frac{\varepsilon}{|\mathbf{s}-\mathbf{t}|^{1 / 2}}\right)^{d} \sigma(d \mathbf{s}),
\end{aligned}
$$


where

$$
Q_{7} \triangleq Q_{6} \wedge\left(4 e \pi c_{2}\right)^{-d / 2}
$$

One can directly check that $Q_{6} \geqslant(2 / e \pi)^{d / 2}$; the result follows from Eq. (5.4).

\section{Proof of the upper bound: Part 3}

Recall the notation of $\S 4$. In the previous section, we found a prediction estimate for $J_{1}$ in terms of the filtration $\mathcal{F}$. The choice of $\mathcal{F}$ was made to accommodate the partial order $\succ_{(1)}$ used in the definition of $J_{1}$. In this section, we wish to provide a prediction estimate for $J_{2}$. Now, the relevant partial order is $\succ_{(2)}$. The filtration $\mathcal{G}$ is designed exactly to provide the analogue of $\mathcal{F}$ in $\S 5$. As in $\S 5$, it all begins with a path decomposition result. Recall from $\S 5$ that $\dot{B}$ denotes $(2, d)$-white noise.

Lemma 6.1. Fix some $\mathbf{t} \in\left[0, \infty\left[{ }^{2}\right.\right.$. For all $\mathbf{s} \succ_{(2)} \mathbf{t}$, define

$$
V\left(s^{(2)}\right) \triangleq B\left(t^{(1)}, s^{(2)}\right)-\frac{s^{(2)}}{t^{(2)}} B(\mathbf{t})
$$

and

$$
U(\mathbf{s}) \triangleq \dot{B}\left(\left[t^{(1)}, s^{(1)}\right] \times\left[0, s^{(2)}\right]\right) .
$$

Then $U$ and $V$ are mutually independent from each other as well as from $\mathcal{G}(\mathbf{t})$. Finally, as a process in $\mathbf{s} \succ_{(2)} \mathbf{t}$,

$$
B(\mathbf{s})=V\left(s^{(2)}\right)+U(\mathbf{s})+\frac{s^{(2)}}{t^{(2)}} B(\mathbf{t}) .
$$

Proof. As was the case with Lemma 5.1, we give a proof based on Cěntsov's white noise construction of $B$; cf. [4]. Elementary considerations show us that

$$
B(\mathbf{s})=B\left(t^{(1)}, s^{(2)}\right)+\dot{B}\left(\left[t^{(1)}, s^{(1)}\right] \times\left[0, s^{(2)}\right]\right),
$$

and the second term is independent of the first as well as $\mathcal{G}(\mathbf{t})$. The rest follows from covariance calculations.

Remark 6.1.1. It is worth-while to point out that the process $\left(V\left(s^{(2)}\right) ; s^{(2)} \in\right.$ $\left.\left[0, t^{(2)}\right]\right)$ of Lemma 6.1 is a Brownian bridge on $\left[0, t^{(2)}\right]$, pinned at 0 at at both ends.

With the decomposition under our belt, we can state and prove the main result of this section. This is the correct analogue of Lemma 5.2.

Lemma 6.2. Fix $\mathbf{t} \in\left[0, \infty\left[{ }^{2}, \sigma \in \mathcal{P}(E), M>0, a \in[-M, M]^{d}\right.\right.$ and $\varepsilon>0$. Then, a.s.,

$$
\mathbb{E}\left[J_{2} \mid \mathcal{G}(\mathbf{t})\right] \geqslant c_{6} \mathbb{1}\left\{\mathbf{t} \in \mathcal{H}_{\varepsilon}^{a}\right\} \int_{\mathbf{s} \succ_{(2)} \mathbf{t}}\left(1 \wedge \frac{\varepsilon}{|\mathbf{s}-\mathbf{t}|^{1 / 2}}\right)^{d} \sigma(d \mathbf{s}),
$$

where

$$
c_{6} \triangleq\left(\frac{2}{\pi}\right)^{d}\left(1 \vee 2^{3 / 2} c_{2}\right)^{-d / 2} \exp \left(-d\left(M^{2}+1\right)\right) .
$$

Proof. Recall Eqs. (4.1) and (4.2). By Lemma 6.1,

$$
J_{2}=\int_{\mathbf{s} \succ(2)} \mathbb{t}\left\{\left|V\left(s^{(2)}\right)+\frac{s^{(2)}}{t^{(2)}} B(\mathbf{t})+U(\mathbf{t})-a\right| \leqslant \varepsilon\right\} \sigma(d \mathbf{s}) .
$$


Therefore, on $\left(\mathbf{t} \in \mathcal{H}_{\varepsilon}^{a}\right)$,

$$
\begin{aligned}
J_{2} & \geqslant \int_{\mathbf{s} \succ(2)} \mathbf{t}\left\{\left|V\left(s^{(2)}\right)+U(\mathbf{s})-\frac{s^{(2)}}{t^{(2)}} a\right| \leqslant \varepsilon-\frac{s^{(2)}}{t^{(2)}} \frac{\varepsilon}{2}\right\} \sigma(d \mathbf{s}) \\
& \geqslant \int_{\mathbf{s} \succ_{(2)} \mathbf{t}} \mathbb{1}\left\{\left|V\left(s^{(2)}\right)+U(\mathbf{s})-\frac{s^{(2)}}{t^{(2)}} a\right| \leqslant \varepsilon / 2\right\} \sigma(d \mathbf{s}) .
\end{aligned}
$$

By the asserted independence of Lemma 6.1, a.s.,

$$
\mathbb{E}\left[J_{2} \mid \mathcal{G}(\mathbf{t})\right] \geqslant \mathbb{1}\left\{\mathbf{t} \in \mathcal{H}_{\varepsilon}^{a}\right\} \int_{\mathbf{s} \succ(2)} \mathbf{t}\left(\left|V\left(s^{(2)}\right)+U(\mathbf{s})-\frac{s^{(2)}}{t^{(2)}} a\right| \leqslant \varepsilon / 2\right) \sigma(d \mathbf{s}) .
$$

Recall from Lemma 6.1 that $U$ and $V$ are independent. Their construction reveals that they are both Gaussian with mean 0, i.i.d. coordinates with coordinate-wise variance given by the following: for all $1 \leqslant i \leqslant d$,

$$
\begin{aligned}
\operatorname{Var}\left(V^{(i)}\left(s^{(2)}\right)\right) & =\frac{s^{(2)} t^{(1)}}{t^{(2)}}\left(t^{(2)}-s^{(2)}\right), \\
\operatorname{Var}\left(U^{(i)}(\mathbf{s})\right) & =s^{(2)}\left(s^{(1)}-t^{(1)}\right) .
\end{aligned}
$$

Hence, by (6.1), the following holds a.s.:

$\mathbb{E}\left[J_{2} \mid \mathcal{G}(\mathbf{t})\right]$

$$
\geqslant \mathbb{1}\left\{\mathbf{t} \in \mathcal{H}_{\varepsilon}^{a}\right\} \int_{\mathbf{s} \succ(2)} \mathbf{t} P\left(\left|\Gamma_{5}-\frac{s^{(2)}}{t^{(2)}} a\right| \leqslant \frac{\varepsilon}{2 \sqrt{\operatorname{Var}\left(V^{(1)}\left(s^{(2)}\right)\right)+\operatorname{Var}\left(U^{(1)}(\mathbf{s})\right)}}\right) \sigma(d \mathbf{s}),
$$

where $\Gamma_{5}$ is a $d$-dimensional random vector whose coordinates are i.i.d. standard Gaussians. From (6.2), it follows that for all $\mathbf{t}, \mathbf{s}$ in question,

$$
\operatorname{Var}\left(V^{(1)}\left(s^{(2)}\right)\right)+\operatorname{Var}\left(U^{(1)}(\mathbf{s})\right) \leqslant 2 c_{2}|\mathbf{s}-\mathbf{t}|
$$

Therefore, with probability one,

$$
\mathbb{E}\left[J_{2} \mid \mathcal{G}(\mathbf{t})\right] \geqslant \mathbb{1}\left\{\mathbf{t} \in \mathcal{H}_{\varepsilon}^{a}\right\} \int_{\mathbf{s} \succ(2)} \mathbf{t} \mathbb{P}\left(\left|\Gamma_{5}-\frac{s^{(2)}}{t^{(2)}} a\right| \leqslant \frac{\varepsilon}{2 \sqrt{2 c_{2}}|\mathbf{s}-\mathbf{t}|^{1 / 2}}\right) \sigma(d \mathbf{s}) .
$$

Temporarily, let

$$
\begin{aligned}
& \eta \triangleq \frac{\varepsilon}{2 \sqrt{2 c_{2}}|\mathbf{s}-\mathbf{t}|^{1 / 2}}, \\
& \delta \triangleq \frac{s^{(2)}}{t^{(2)}}, \\
& P \triangleq \mathbb{P}\left(\left|\Gamma_{5}-\delta a\right| \leqslant \eta\right) .
\end{aligned}
$$

Clearly,

$$
\begin{aligned}
P & =\prod_{j=1}^{d} \int_{\delta a-\eta}^{\delta a+\eta} \frac{e^{-u^{2} / 2}}{\sqrt{2 \pi}} d u \\
& \geqslant\left(\frac{2}{\pi}\right)^{d} \eta^{d} \exp \left(-\frac{\sum_{j=1}^{d}\left(\delta a^{(j)}-\eta\right)^{2} \vee \sum_{k=1}^{d}\left(\delta a^{(k)}+\eta\right)^{2}}{2}\right) \\
& \geqslant\left(\frac{2}{\pi}\right)^{d} \eta^{d} \exp \left(-d|a|-d \eta^{2}\right)
\end{aligned}
$$


We have used the fact that, in this regime, $\delta \leqslant 1$ and $(a \pm \eta)^{2} \leqslant 2\left(a^{2}+\eta^{2}\right)$. Since $|a| \leqslant M$, if $\eta \leqslant 1$

$$
P \geqslant\left(\frac{2}{\pi}\right)^{d} e^{-d\left(M^{2}+1\right)} 2^{-3 d / 2} c_{2}^{-d / 2} \frac{\varepsilon^{d}}{|\mathbf{s}-\mathbf{t}|^{d / 2}} .
$$

On the other hand, if $\eta>1$, a similar analysis shows that

$$
\begin{aligned}
P & \geqslant \mathbb{P}\left(\left|\Gamma_{5}-\delta a\right| \leqslant 1\right) \\
& \geqslant\left(\frac{2}{\pi}\right)^{d} e^{-d\left(M^{2}+1\right)} .
\end{aligned}
$$

Combining this with (6.4), we obtain the following:

$$
P \geqslant\left(\frac{2}{\pi}\right)^{d} e^{-d\left(M^{2}+1\right)}\left(1 \vee 2^{3 / 2} c_{2}\right)^{-d / 2}\left(1 \wedge \frac{\varepsilon}{|\mathbf{s}-\mathbf{t}|^{1 / 2}}\right)^{d} .
$$

Eq. (6.3) is now easily seen to imply the desired result.

\section{Proof of the upper bound: Part 4}

We are finally ready to put things together to obtain the proof of the upper bound of Theorem 2.1. Fix $\varepsilon>0, a \in[-M, M]^{d}$, and define

$$
T_{\varepsilon}^{(1)} \triangleq \inf (s>0: \exists t>0 \text { such that }(s, t) \in E \text { and }|B(s, t)-a| \leqslant \varepsilon) .
$$

By compactness and sample path continuity, $T_{\varepsilon}^{(1)}$ is a random variable. Next, we define (also a random variable):

$$
T_{\varepsilon}^{(2)} \triangleq \inf \left(t>0:\left(T_{\varepsilon}^{(1)}, t\right) \in E \text { and }\left|B\left(T_{\varepsilon}^{(1)}, t\right)-a\right| \leqslant \varepsilon\right) .
$$

As usual, we have implicitly defined $\inf \varnothing \triangleq \infty$ and we extend the temporal domain of $B$ such that $B(\infty, \infty) \triangleq \Delta$, a cemetery state. In such a way - and recalling (4.2) - we can now note that

$$
\mathcal{H}_{\varepsilon}^{a} \neq \varnothing \Longleftrightarrow\left|B\left(\mathbf{t}_{\varepsilon}\right)-a\right| \leqslant \varepsilon \Longleftrightarrow \mathbf{t}_{\varepsilon} \in \mathcal{H}_{\varepsilon}^{a} .
$$

In accordance with notation set in $\S 1, \mathbf{t}_{\varepsilon} \triangleq\left(T_{\varepsilon}^{(1)}, T_{\varepsilon}^{(2)}\right)$. By standard real variable arguments and Proposition 4.2(iii) and (iv), the null sets in Lemmas 5.2 and 6.2 can be chosen independently of the choice of $\mathbf{t}$. In particular, picking $\mathbf{t}=\mathbf{T}_{\varepsilon}$, we obtain the following from (7.1): a.s.

$$
\sup _{\mathbf{t} \in\left[0, \infty\left[^{2}\right.\right.} \mathbb{E}\left[J_{1} \mid \mathcal{F}(\mathbf{t})\right] \geqslant c_{5} \mathbb{1}\left\{\mathcal{H}_{\varepsilon}^{a} \neq \varnothing\right\} \int_{\mathbf{s} \succ(1)}\left(1 \wedge \frac{\varepsilon}{\left|\mathbf{s}-\mathbf{t}_{\varepsilon}\right|^{1 / 2}}\right)^{d} \sigma(d \mathbf{s})
$$

and

$$
\sup _{\mathbf{t} \in\left[0, \infty\left[^{2}\right.\right.} \mathbb{E}\left[J_{2} \mid \mathcal{G}(\mathbf{t})\right] \geqslant c_{6} \mathbb{1}\left\{\mathcal{H}_{\varepsilon}^{a} \neq \varnothing\right\} \int_{\mathbf{s} \succ(2)}\left(1 \wedge \frac{\varepsilon}{\left|\mathbf{s}-\mathbf{t}_{\varepsilon}\right|^{1 / 2}}\right)^{d} \sigma(d \mathbf{s}) .
$$

It is high time we picked $\sigma \in \mathcal{P}(E)$ judiciously. Define $\sigma \triangleq \sigma_{\varepsilon}$ to be the distribution of $\mathbf{t}_{\varepsilon}$, conditioned on the measurable event $\left(\mathcal{H}_{\varepsilon}^{a} \neq \varnothing\right)$. Clearly, $\sigma_{\varepsilon} \in \mathcal{P}(E)$. 
Therefore, (7.2) and (7.3) hold for this $\sigma_{\varepsilon}$ replacing $\sigma$ everywhere. Squaring (7.2) and (7.3) - for this $\sigma_{\varepsilon}$ - and taking expectations, we obtain the following:

$$
\begin{aligned}
& \mathbb{E}\left[\sup _{\mathbf{t} \in\left[0, \infty\left[\left[^{2}\right.\right.\right.} \mathbb{E}\left[J_{1} \mid \mathcal{F}(\mathbf{t})\right]\right]^{2} \geqslant c_{5}^{2} \mathbb{P}\left(\mathcal{H}_{\varepsilon}^{a} \neq \varnothing\right) \mathbb{E}\left[\left(Z_{1}\right)^{2} \mid \mathcal{H}_{\varepsilon}^{a} \neq \varnothing\right], \\
& \mathbb{E}\left[\sup _{\mathbf{t} \in\left[0, \infty\left[^{2}\right.\right.} \mathbb{E}\left[J_{2} \mid \mathcal{G}(\mathbf{t})\right]\right]^{2} \geqslant c_{6}^{2} \mathbb{P}\left(\mathcal{H}_{\varepsilon}^{a} \neq \varnothing\right) \mathbb{E}\left[\left(Z_{2}\right)^{2} \mid \mathcal{H}_{\varepsilon}^{a} \neq \varnothing\right],
\end{aligned}
$$

where, for $i=1,2$,

$$
Z_{i} \triangleq \int_{\mathbf{s} \succ(i)} \mathbf{t}_{\varepsilon}\left(1 \wedge \frac{\varepsilon}{\left|\mathbf{s}-\mathbf{t}_{\varepsilon}\right|^{1 / 2}}\right)^{d} \sigma_{\varepsilon}(d \mathbf{s}) .
$$

By the definition of $\sigma_{\varepsilon}$ and the Cauchy-Schwartz inequality, for $i=1,2$,

$$
\begin{aligned}
\sqrt{\mathbb{E}\left[\left(Z_{i}\right)^{2} \mid \mathcal{H}_{\varepsilon}^{a} \neq \varnothing\right]} & \geqslant \mathbb{E}\left[Z_{i} \mid \mathcal{H}_{\varepsilon}^{a} \neq \varnothing\right] \\
& =\int_{\mathbf{s} \succ(i)} \int_{\mathbf{t}}\left(1 \wedge \frac{\varepsilon}{|\mathbf{s}-\mathbf{t}|^{1 / 2}}\right)^{d} \sigma_{\varepsilon}(d \mathbf{s}) \sigma_{\varepsilon}(d \mathbf{t}) .
\end{aligned}
$$

Using $(a+b)^{2} \leqslant 2\left(a^{2}+b^{2}\right)$ together with real variable arguments, we obtain:

$$
\begin{aligned}
\mathbb{E}\left[\left(Z_{1}\right)^{2}+\left(Z_{2}\right)^{2} \mid \mathcal{H}_{\varepsilon}^{a} \neq \varnothing\right] & \geqslant \sum_{i=1}^{2}\left\{\int_{\mathbf{s} \succ(i)} \mathbf{t}\left(1 \wedge \frac{\varepsilon}{|\mathbf{t}-\mathbf{s}|^{1 / 2}}\right)^{d} \sigma_{\varepsilon}(d \mathbf{s}) \sigma_{\varepsilon}(d \mathbf{t})\right\}^{2} \\
& \geqslant \frac{1}{2}\left\{\sum_{i=1}^{2} \iint_{\mathbf{s} \succ(i)}\left(1 \wedge \frac{\varepsilon}{|\mathbf{t}-\mathbf{s}|^{1 / 2}}\right)^{d} \sigma_{\varepsilon}(d \mathbf{s}) \sigma_{\varepsilon}(d \mathbf{t})\right\}^{2} \\
& \geqslant \frac{1}{8}\left\{\iint\left(1 \wedge \frac{\varepsilon}{|\mathbf{t}-\mathbf{s}|^{1 / 2}}\right)^{d} \sigma_{\varepsilon}(d \mathbf{s}) \sigma_{\varepsilon}(d \mathbf{t})\right\}^{2}
\end{aligned}
$$

On the other hand, since $0 \leqslant J_{i} \leqslant 1$, Proposition 4.2(i) and (ii) imply that

$$
\begin{aligned}
& \mathbb{E}\left[\sup _{\mathbf{t} \in\left[0, \infty\left[^{2}\right.\right.} \mathbb{E}\left[J_{1} \mid \mathcal{F}(\mathbf{t})\right]\right]^{2} \leqslant 16 \mathbb{E}\left[J_{1}\right]^{2}, \\
& \mathbb{E}\left[\sup _{\mathbf{t} \in\left[0, \infty\left[^{2}\right.\right.} \mathbb{E}\left[J_{2} \mid \mathcal{G}(\mathbf{t})\right]\right]^{2} \leqslant 16 \mathbb{E}\left[J_{2}\right]^{2} .
\end{aligned}
$$

Furthermore, it follows immediately from (4.1) that, for $i=1,2, J_{i} \leqslant I_{\varepsilon}^{a}\left(\sigma_{\varepsilon}\right)$. Therefore, from Lemma 3.2 we see that, for $i \in\{1,2\}$,

$$
\mathbb{E}\left(J_{1}^{2}+J_{2}^{2}\right) \leqslant 2 c_{4} \varepsilon^{d} \iint\left(1 \wedge \frac{\varepsilon}{|\mathbf{s}-\mathbf{t}|^{1 / 2}}\right)^{d} \sigma_{\varepsilon}(d \mathbf{s}) \sigma_{\varepsilon}(d \mathbf{t}) .
$$

Eqs. (7.4), (7.5), (7.6) and (7.7) together imply the following:

$$
\mathbb{P}\left(\mathcal{H}_{\varepsilon}^{a} \neq \varnothing\right) \leqslant \frac{256 c_{4}}{\left(c_{5}^{2} \wedge c_{6}^{2}\right)}\left[\iint\left(\frac{1}{\varepsilon} \wedge \frac{1}{|\mathbf{s}-\mathbf{t}|^{1 / 2}}\right)^{d} \sigma_{\varepsilon}(d \mathbf{s}) \sigma_{\varepsilon}(d \mathbf{t})\right]^{-1} .
$$

Let us fix a $\lambda>0$. Clearly, for all $\varepsilon<\lambda^{2}$,

$$
\mathbb{P}\left(\mathcal{H}_{\varepsilon}^{a} \neq \varnothing\right) \leqslant \frac{256 c_{4}}{\left(c_{5}^{2} \wedge c_{6}^{2}\right)}\left[\iint_{|\mathbf{s}-\mathbf{t}| \geqslant \lambda}|\mathbf{s}-\mathbf{t}|^{-d / 2} \sigma_{\varepsilon}(d \mathbf{s}) \sigma_{\varepsilon}(d \mathbf{t})\right]^{-1} .
$$


Now we can finish the proof. Since $\left(\sigma_{\varepsilon} ; 0<\varepsilon<\lambda^{2}\right)$ is a sequence of probability measures all living on the compact set $E$, Prohorov's theorem allows us to extract a sequence $\varepsilon_{k}$, such that

(a) $\lim _{k \rightarrow \infty} \varepsilon_{k}=0$;

(b) $\sigma_{\varepsilon_{k}}$ converges weakly to some $\sigma_{0} \in \mathcal{P}(E)$.

Along this sequence, we can use Eq. (7.8) and the Portmanteau theorem of weak convergence theory, to see that

$$
\limsup _{k \rightarrow \infty} \mathbb{P}\left(\mathcal{H}_{\varepsilon_{k}}^{a} \neq \varnothing\right) \leqslant \frac{256 c_{4}}{\left(c_{5}^{2} \wedge c_{6}^{2}\right)}\left[\iint_{|\mathbf{s}-\mathbf{t}| \geqslant \lambda}|\mathbf{s}-\mathbf{t}|^{-d / 2} \sigma_{0}(d \mathbf{s}) \sigma_{0}(d \mathbf{t})\right]^{-1} .
$$

Path continuity and compactness reveal that

$$
\limsup _{k \rightarrow \infty} \mathbb{P}\left(\mathcal{H}_{\varepsilon_{k}}^{a} \neq \varnothing\right)=\mathbb{P}(a \in B(E)) .
$$

By the monotone convergence theorem (letting $\lambda \downarrow 0$ ),

$$
\mathbb{P}(a \in B(E)) \leqslant \frac{256 c_{4}}{\left(c_{5}^{2} \wedge c_{6}^{2}\right)} \operatorname{Cap}_{d / 2}(E)
$$

The upper bound in Theorem 2.1 follows.

\section{EPILOGUe}

We conclude with some scattered remarks and problems.

8.1. Upon closer examination of its proof, Theorem 2.1 can be extended to the following:

Theorem 8.1. In the notation of Theorem 2.1, for all $0<\varepsilon<M$,

$$
\begin{aligned}
& A_{1}\left[\inf _{\sigma \in \mathcal{P}(E)} \iint\left(\varepsilon \vee|\mathbf{s}-\mathbf{t}|^{1 / 2}\right)^{-d} \sigma(d \mathbf{s}) \sigma(d \mathbf{t})\right]^{-1} \\
& \quad \leqslant \mathbb{P}(\operatorname{dist}[B(E), a] \leqslant \varepsilon) \\
& \quad \leqslant A_{2}\left[\inf _{\sigma \in \mathcal{P}(E)} \iint\left(\varepsilon \vee|\mathbf{s}-\mathbf{t}|^{1 / 2}\right)^{-d} \sigma(d \mathbf{s}) \sigma(d \mathbf{t})\right]^{-1},
\end{aligned}
$$

where dist $[B(E), a]$ denotes the Hausdorff distance between $B(E)$ and $\{a\}$.

8.2. Suppose $\left(B(\mathbf{s}): \mathbf{s} \in\left[0, \infty\left[{ }^{N}\right)\right.\right.$ is $(N, d)$-Brownian sheet. Is it true that for all compact sets $E \subset\left[0, \infty\left[{ }^{N}, \mathbb{E}\left[\operatorname{Leb}_{d}(B(E))\right]>0\right.\right.$ if and only if $\operatorname{Cap}_{d / 2}(E)>0$ ? $\left(\operatorname{Cap}_{d / 2}(E)\right.$ is defined in complete analogy to the $N=2$ case; see Introduction.) While I have not checked all of the details, it seems that the methods of this paper should extend to values of $N$ higher than $N=2$. Likewise, Theorem 2.1 seems to have its multi-parameter extensions for $N>2$. The only possible source of difficulty is this: when $N=2$, we needed two partial orders. Namely, $\succ_{(1)}$ and $\succ_{(2)}$. Each one corresponds to a fundamentally different path decomposition and each path decomposition requires its own analysis. In general, we need $2^{N-1}$ such path decompositions. It is conceivable that some of them may yield objects which are not easy to analyse. (I do not think that this is the case, however.) 
8.3. Suppose $X$ and $Y$ are two independent $d$-dimensional Brownian motions. As in $\S 1$, we define additive Brownian motion $Z \triangleq X \oplus Y$ by

$$
Z(s, t)=X(s)+Y(t), \quad(s, t) \in\left[0, \infty\left[^{2} .\right.\right.
$$

The proof of the following is similar to - though easier than - that of Theorem 2.1. We omit the details.

Theorem 8.2. Fix some $M>0$, a compact set $E \subset\left[0, \infty\left[{ }^{2}\right.\right.$, and $a \in[-M, M]^{d}$. Then, there exist constants $A_{5}$ and $A_{6}$ which depend only only $c_{1}(E), c_{2}(E), M$ and $d$, such that

$$
A_{5} \operatorname{Cap}_{d / 2}(E) \leqslant \mathbb{P}(a \in Z(E)) \leqslant A_{6} \operatorname{Cap}_{d / 2}(E) .
$$

We have already mentioned that this problem is due to J.-P. Kahane. See [7, Chapter 16] for partial results. Furthermore, T.S. Salisbury has another proof of Theorem 8.2. (This material is to be published.) The latter uses Markovian techniques while we use Gaussian ones. Each method has its own advantages in its applicability to processes other than the Brownian sheet.

8.4. Is there a way to combine the question addressed in this paper with the potential theory of [10]? To be more precise, is there an exact capacitary condition on $E \times \Theta$ for $\mathbb{E}\left[\operatorname{Leb}_{d}(B(E) \cap \Theta)\right]$ to be strictly positive? Here, $E \subset[0, \infty[2$ and $\Theta \subset \mathbb{R}^{d}$ are both compact. In its simplest setting $(N=1$, i.e., Brownian motion), there seems to be nontrivial connections - see [8] and [13]. For a general approach to parabolic potential theory, see [2].

\section{REFERENCES}

1. R. J. Adler, The Geometry of Random Fields, Wiley, London, 1981. MR 82h:60103

2. H. Ben Saud and K. Jenßen, A characterization of parabolic potential theory, Math. Ann., 272 (1985), 281-289.

3. I. Benjamini, R. Pemantle and Y. Peres, Martin capacity for Markov chains, Ann. Prob., 23 (1995), 1332-1346. MR 96g:60098

4. N. N. Cěntsov, Wiener random fields depending on several parameters, Dokl. Akad. Nauk S.S.S.R. (NS), 106 (1956), 607-609.

5. R. C. Dalang and J. B. Walsh, Geography of the level sets of the Brownian sheet, Prob. Th. Rel. Fields, 96 (1993), 153-176. MR 98f:60093

6. P. Imkeller, Two-parameter Martingales and Their Quadratic Variation, Lecture Notes in Mathematics, vol. 1308, Springer, New York, 1988. MR 89e:60098

7. J.-P. Kahane, Some Random Series of Functions, Cambridge University Press, Cambridge, 1985. MR 87m:60119

8. R. Kaufman and J. M. Wu, Parabolic Potential Theory, J. Diff. Eq., 43, (1982), 204-234. MR 83d:31006

9. D. Khoshnevisan Some polar sets for the Brownian sheet, Sém. de Prob., XXXI, Lecture Notes in Mathematics, vol. 1655, pp. 190-197, 1997. CMP 98:03

10. D. Khoshnevisan and Z. Shi, Brownian sheet and capacity, Preprint, 1997

11. S. Orey and W. E. Pruitt, Sample functions of the N-parameter Wiener process, Ann. Prob., 1 (1973), 138-163. MR 49:11646

12. E. M. Stein, Singular Integrals and Differentiability Properties of Functions, Princeton University Press, Fifth Edition, 1986. MR 44:7280

13. Y. Xiao, Hitting probabilities and polar sets for fractional Brownian motion, Preprint, 1997.

Department of Mathematics, University of Utah, Salt Lake City, Utah 84112

E-mail address: davar@math.utah.edu 